\title{
Catalytic CO Oxidation over Au Nanoparticles Loaded Nanoporous Nickel Phosphate Composite
}

\author{
Xiaonan Leng, ${ }^{1}$ Dantong Zhou, ${ }^{1}$ Tong Gao, ${ }^{1}$ Zhi Chen, ${ }^{1,2}$ and Qiuming Gao ${ }^{2,3}$ \\ ${ }^{1}$ College of Materials Science and Engineering, China Jiliang University, No. 258 Xueyuan Street, Xiasha Higher Education District, \\ Hangzhou, Zhejiang 310018, China \\ ${ }^{2}$ State Key Laboratory of High Performance Ceramics and Superfine Microstructures, Shanghai Institute of Ceramics, \\ Chinese Academy of Sciences, Shanghai 200050, China \\ ${ }^{3}$ Key Laboratory of Bio-Inspired Smart Interfacial Science and Technology of Ministry of Education, \\ School of Chemistry and Environment, Beihang University, Beijing 100191, China
}

Correspondence should be addressed to Zhi Chen; zchen0@gmail.com and Qiuming Gao; qmgao@buaa.edu.cn

Received 18 September 2014; Revised 30 November 2014; Accepted 2 December 2014

Academic Editor: Ashok K. Sundramoorthy

Copyright (C) 2015 Xiaonan Leng et al. This is an open access article distributed under the Creative Commons Attribution License, which permits unrestricted use, distribution, and reproduction in any medium, provided the original work is properly cited.

\begin{abstract}
$\mathrm{Au} /$ nickel phosphate-5 (Au/VSB-5) composite with the noble metal loading amount of $1.43 \mathrm{wt}$.\% is prepared by using microporous VSB-5 nanocrystals as the support. Carbon monoxide (CO) oxidation reaction is carried out over the sample with several catalytic cycles. Complete conversion of $\mathrm{CO}$ is achieved at $238^{\circ} \mathrm{C}$ over the catalyst at the first catalytic cycle. The catalytic activity improved greatly at the second cycle with the complete conversion fulfilled at $198^{\circ} \mathrm{C}$ and preserved for the other cycles. A series of experiments such as X-ray diffraction (XRD), high resolution transmission electron microscopy (HRTEM), ultraviolet-visible (UV-vis) spectroscopy, and X-ray photoelectron spectroscopy (XPS) are carried out to characterize the catalysts before and after the reaction to study the factors influencing this promotion at the second cycle.
\end{abstract}

\section{Introduction}

Air pollution such as carbon monoxide (CO) contamination is a deteriorating environmental issue in the world which strongly affects the human activity. The catalytic removal of the air pollutant on nanosized catalyst is one of the effective strategies for the pollution control. Gold nanoparticles have attracted a rapid growing interest and played a vital role in many fields such as therapeutics, electronics, and pollution controls in which a new kind of gold rush has been developed [1]. Among the reported gold particles, supported ones have become attractive since they have high catalytic activities over the oxygen-transfer reaction at near-ambient temperature [25]. They have wide applications in series of reactions such as organic syntheses and waste gas disposals (CO and nitric oxide (NO) elimination) [6]. The performance of gold catalyst on the oxidation of $\mathrm{CO}$ has a close relationship with particle size and band valence of the gold, nature of the support, and preparation method [7-10]. In addition, the oxidation elimination of $\mathrm{CO}$, except for the industrial demands on resources and environmental concerns, may be used as a model reaction to evaluate the catalytic performances of new materials serving as catalysts $[7,8]$. Generally, a high activity for the oxidation of $\mathrm{CO}$ at a low temperature can be acquired when gold nanoparticles are well dispersed on semiconductor oxides such as $\mathrm{Fe}_{2} \mathrm{O}_{3}, \mathrm{TiO}_{2}$, and $\mathrm{CeO}_{2}$.

However, these Au catalysts are unstable and their catalytic activity declines with time due to the aggregation of $\mathrm{Au}$ particles or the adsorption of carbonate which hinders the commercial applications [11]. On the other hand, the amount of gold is limited and the price is high. To overcome these shortcomings, secondary metal was introduced to gold to form bimetallic materials or alloy [11, 12]. Besides, it is a long-term and interesting topic to explore lower loading amount of noble metals with remaining activity and the general approaches are carried on by employing novel substitutes or new preparation methods [13]. Recently, ultra-low loading of $\mathrm{Au}(0.1 \mathrm{wt} . \%)$ on $\mathrm{CeO}_{2}$ was prepared 
by different preparation methods. However, the catalytic activities on these catalysts decreased during the continued $\mathrm{CO}$ oxidation process [14]. Zeolites have been used as another kind of supports for the gold related composite catalysts due to their advantages of high surface area and ion-exchange ability. Small gold nanoparticles could be stabilized inside the micropores [6,15-17]. Interaction between these supports and gold nanoparticles is believed to affect the performance of the catalysts. Efforts have been focused on the preparation of suitable supports to improve the synergetic effects between the supports and gold nanoparticles [18, 19]. However, the exact nature of the active sites and the relevance of the support for the oxidation are still disputable. Zeolite-type metal-organic framework (MOF) was recently introduced as the active host of gold catalyst for the $\mathrm{CO}$ oxidation although the temperature for $50 \%$ conversion of CO was high [20].

Developing a new active support for gold nanoparticles is vital for exploring and applying novel kind of multifunctional Au catalysts. Nickel phosphate VSB-5 is a kind of zeolite-type material posing ordered $1.1 \mathrm{~nm}$ one-dimensional channels framed from $\mathrm{NiO}_{6}$ and $\mathrm{PO}_{4}$ building blocks [21]. New techniques, such as microwave, have been employed to realize the facile synthesis, size control, and high performance $[22,23]$. Other concerns have been focused on functionalizing VSB-5 by isomorphously substituting the nickel in the framework with transitional metal ions such as iron ions, cobalt ions, zirconium ions, and manganese ions or by treating the powders with $\mathrm{NaOH}$ [24-26]. Newly catalytic activities on these materials have been achieved for some vital oxidation reactions such as oxidation of phenol and alcohol. Supported Ag/VSB-5 nanocomposite has also been fabricated based on the as-synthesized nanocrystals which showed good performance for the oxidation of styrene [27]. Herein, we report the preparation of Au/VSB-5 nanocomposites with low Au quantity based on the VSB- 5 nanocrystals from the point of broadening the applications of Au and VSB-5 catalysts. The catalytic activity of the composites was evaluated by using the oxidation of $\mathrm{CO}$ as the model reaction at mild temperature.

\section{Experimental}

2.1. Syntheses. VSB-5 nanocrystals were synthesized according to the reported procedure [27]. In a typical experiment, $5.95 \mathrm{~g} \mathrm{NiCl}_{2} \cdot 6 \mathrm{H}_{2} \mathrm{O}$ was dissolved into $80.0 \mathrm{~mL} \mathrm{H}_{2} \mathrm{O}$ and then $1.7 \mathrm{~mL} \mathrm{H}_{3} \mathrm{PO}_{4}(85 \%)$ was added. $10.5 \mathrm{~mL}$ triethylamine was put into the above mixture and the solution was stirred for $30 \mathrm{~min}$. All the reagents were used without further purification. The mixture was transferred into an autoclave and heated at $170^{\circ} \mathrm{C}$ for 6 days. VSB- 5 products were acquired after sufficient washing and drying at $100^{\circ} \mathrm{C}$ for $12 \mathrm{~h}$.

The preparation of Au/VSB-5 nanocomposites was carried out as follows. First, $0.50 \mathrm{~g}$ of the as-synthesized VSB5 was immerged into $40.0 \mathrm{~mL}$ of $\mathrm{HAuCl}_{4}$ solution at a concentration of $2.0 \mathrm{mM}$ and stirred constantly for 2 days. Then, the solid was washed with deionized water for three times and separated by centrifugation. The separated solid was dried at $40^{\circ} \mathrm{C}$ and then was heated under a gas-flowing mixture of $\mathrm{H}_{2}$ and $\mathrm{N}_{2}\left(3 \% \mathrm{H}_{2}\right.$, volume ratio) at $200^{\circ} \mathrm{C}$ for $2 \mathrm{~h}$.

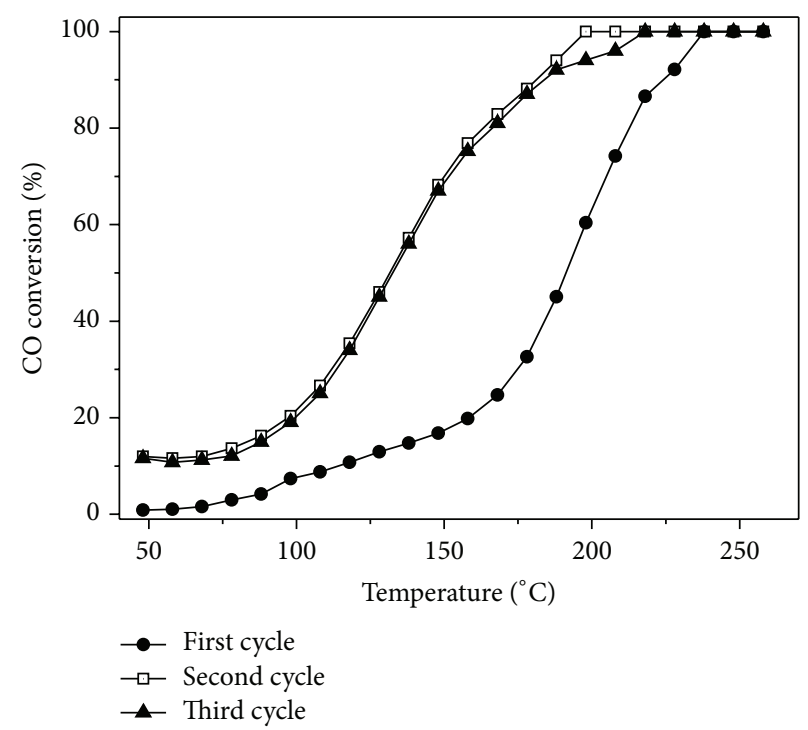

FIGURE 1: CO conversion percentage as a function of temperature for different catalytic cycles over the Au/VSB-5 composite.

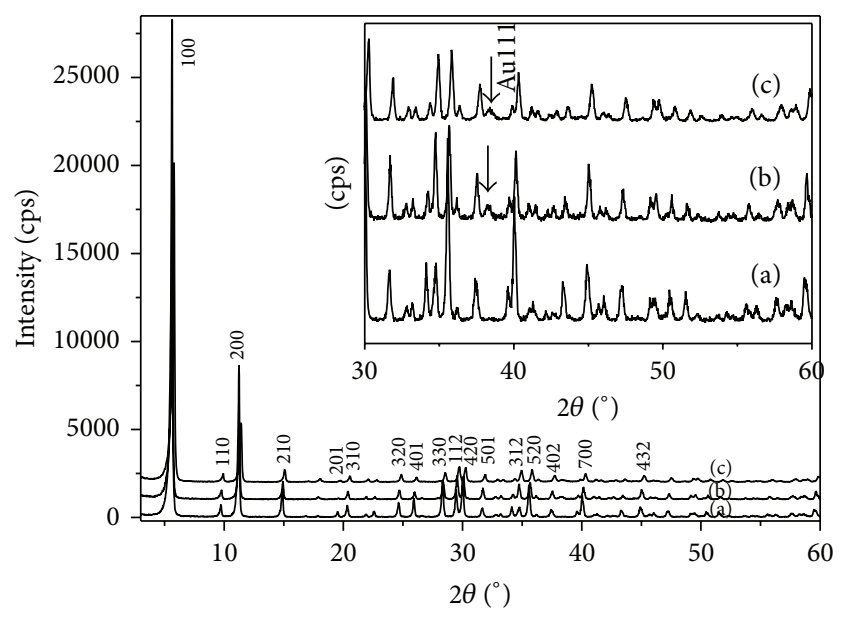

FIGURE 2: XRD patterns of VSB-5 (a) and Au/VSB-5 before (b) and after (c) the catalytic reactions. The inset is the partly enlarged XRD patterns.

At last, the solid product was acquired after cooling down and denoted by Au/VSB-5.

2.2. Characterizations. Powder X-ray diffraction (XRD) pattern analyses were operated on a Rigaku D/max 2200 PC with $\mathrm{Cu} \mathrm{K} \alpha$ radiation. Inductively coupled plasma (ICP) analysis was operated on a Vista Axial CCD Simultaneous ICPAES spectrometer. High resolution transmission electron microscopy (HRTEM) images were taken on a field emission JEM 2010 electron microscope at $200 \mathrm{kV}$. Ultraviolet-visible (UV-vis) diffusing reflectance spectra of the samples were conducted on a Shimadzu UV-3101 instrument equipped with an integrating sphere using $\mathrm{BaSO}_{4}$ as the reference. X-ray photoelectron spectroscopy (XPS) analyses were carried out on a Microlab 310-F Scanning Auger microprobe equipped 
TABLE 1: Comparison of the catalytic performances of Au/VSB-5 and Au@ZIF-8 (the data of Au@ZIF-8 are from [20]).

\begin{tabular}{lccccc}
\hline & Au@ZIF-8 & Au@ZIF-8 & Au@ZIF-8 & Au@ZIF-8 & Au/VSB-5 \\
\hline Au amount (wt.\%) & 0.5 & 1.0 & 1.5 & 2.0 & 1.43 \\
Temperatures of 50\% conversion of CO $\left({ }^{\circ} \mathrm{C}\right)$ & 225 & 200 & 185 & 170 & 132 \\
\hline
\end{tabular}

${ }^{*}$ ZIF-8 (Zn(2-methylimidazole) $\left.)_{2}-8\right)$; VSB-5 (nickel phosphate-5).

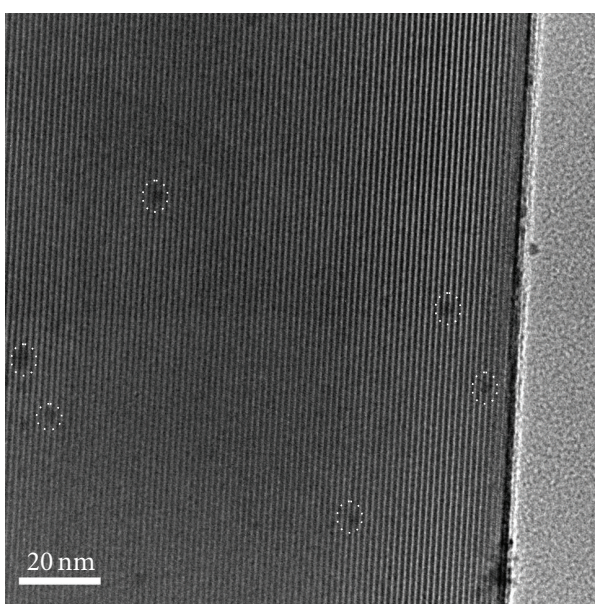

(a)

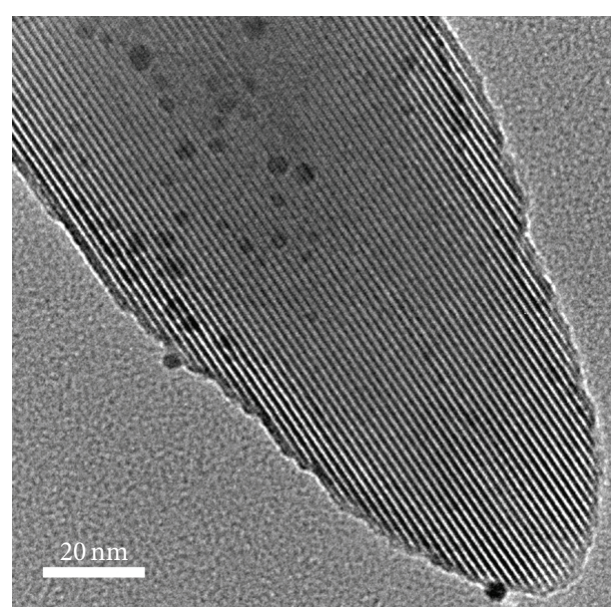

(b)

FIGURE 3: HRTEM image of Au/VSB-5 composite before (a) and after (b) the first catalytic cycle.

with a $150 \mathrm{~W}(15 \mathrm{kV}$ by $10 \mathrm{~mA})$ aluminum $\mathrm{K} \alpha(1486.6 \mathrm{eV})$ anode as the X-ray source, using pass energy of $50 \mathrm{eV}$ with a step size of $0.1 \mathrm{eV}$. The samples were analyzed in the XPS chamber under ultrahigh vacuum $\left(10^{-7} \mathrm{~Pa}\right.$ base pressure). The samples were dried at $40^{\circ} \mathrm{C}$ under vacuum for $8 \mathrm{~h}$ before the XPS measurement. The XPS spectra of Au $4 \mathrm{f}$ were calibrated by using $\mathrm{C}$ 1s spectrum as the standard.

2.3. Catalytic Reactions. Evaluation of the catalyst for $\mathrm{CO}$ oxidation was tested in a fixed-bed quartz tubular reactor with a diameter of $12 \mathrm{~mm} .100 .0 \mathrm{mg}$ of the catalysts was placed in the reactor. The reaction gas $\left(1 \% \mathrm{CO}, 4 \% \mathrm{O}_{2}\right.$, and the balance of $\mathrm{He}$ ) went through the reactor with a flow rate of $50.0 \mathrm{~mL} / \mathrm{min}$, corresponding to space velocity of $30,000 \mathrm{~mL} \cdot \mathrm{h}^{-1} \cdot \mathrm{g}_{\text {cat }}{ }^{-1}$. The concentrations of the outlet $\mathrm{CO}$ after stepwise changes in the reaction temperatures were analyzed with an online gas chromatograph (SP-6890, molecular sieves $13 x$ column) equipped with a thermal conductivity detector (TCD). The conversion of $\mathrm{CO}$ was calculated using the integrated peak area differences between the $\mathrm{CO}$ fed initially and the effluent $\mathrm{CO}$ from the reactor with an accuracy of about $\pm 5 \%$.

\section{Results and Discussions}

The plots of conversion of $\mathrm{CO}$ versus the temperatures are shown in Figure 1. The catalytic reaction occurred at $48^{\circ} \mathrm{C}$ and the complete conversion of $\mathrm{CO}$ was achieved at $238^{\circ} \mathrm{C}$ at the first catalytic cycle. It is interesting to note that the catalytic activity improved greatly at the second cycle. The conversion of $\mathrm{CO}$ reached $12 \%$ at $48^{\circ} \mathrm{C}$ and the complete conversion was fulfilled at $198^{\circ} \mathrm{C}$. The catalytic activity remains for the third catalytic cycle although slight decrease is also observed. The loading amount of Au was 1.43 wt.\% based on the ICP analyses. This full conversion temperature is lower than that of 2 wt.\% Au loading of Au@ZIF-8 which has 50\% conversion of $\mathrm{CO}$ at $185^{\circ} \mathrm{C}[20]$. The catalytic performances of these two catalysts are listed in Table 1.

A series of experiments were carried out to characterize the catalysts before and after the reaction to understand the detailed factors for this promotion at the second cycle. Powder XRD patterns (Figure 2) show that the diffraction peaks of the Au/VSB-5 composites resemble that of the pure VSB-5, indicating that the structure of the host was preserved during the synthetic processes. Intensity of some peaks decreased for the Au/VSB-5 compared with that of the as-synthesized VSB-5. The assembly of Au in the channels of VSB-5 may decrease the scattering ratios between the walls of the host channels and Au guests, which may result in the intensity decrease of some diffraction peaks, such as (100), (110), (200), (210), (310), (320), (410), (330), (112), and (420). Similar phenomena were observed on Ag/VSB-5 [27], Ag/VSB-1 [28], and Ag-ZnS/VSB-1 [29]. The gold nanoparticles were crystalline and an obvious Au (111) diffraction peak of cubic $\mathrm{Au}$ (JCPDS number 89-3697) for the Au/VSB-5 before and after the catalytic reaction was directly observed in Figure 2. The broadened FWHM (full width at half-maximum) of 

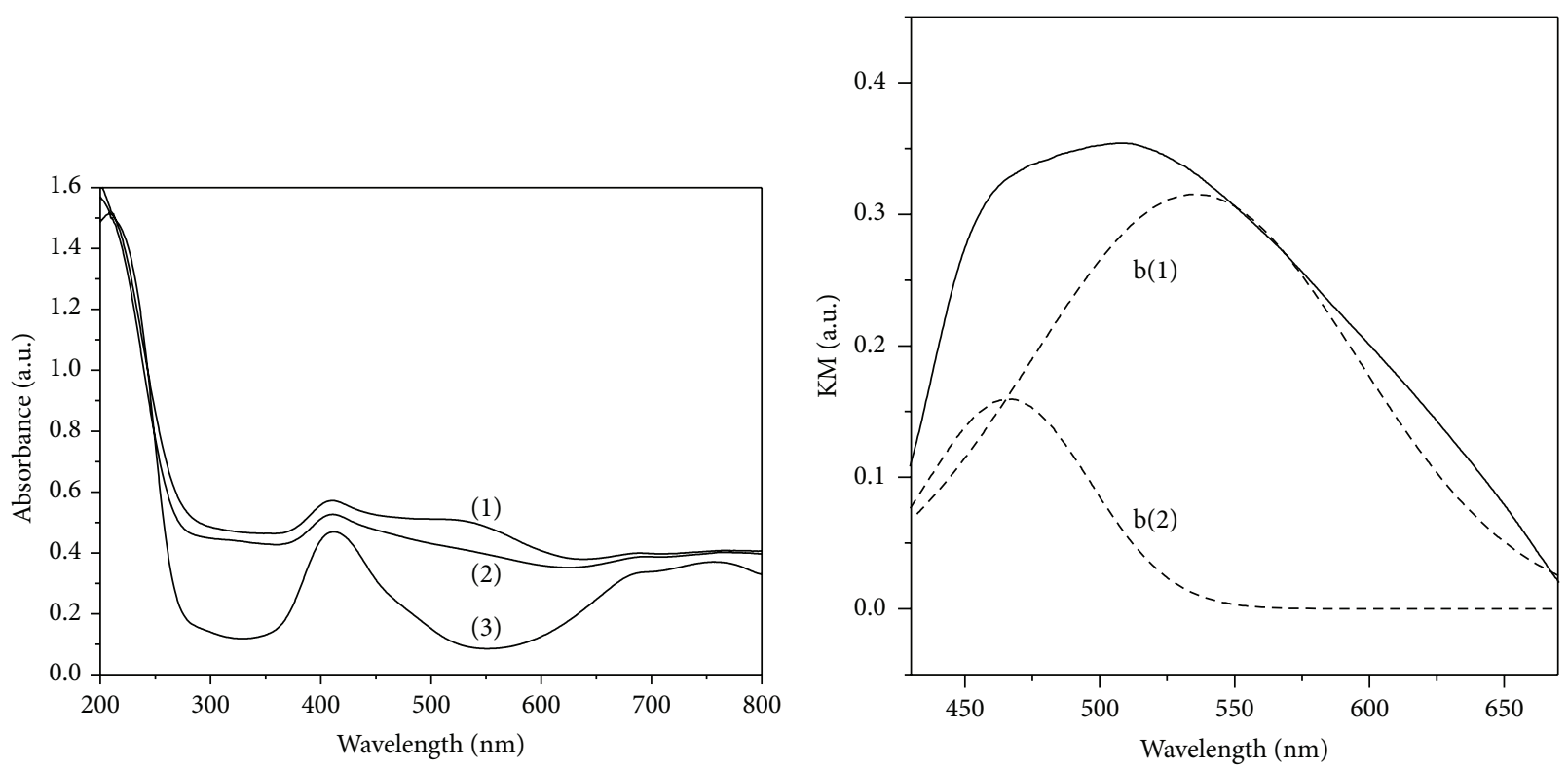

(1) Au/VSB-5 after catalytic reaction

(2) Au/VSB-5 before catalytic reaction

(3) VSB-5

(a)

(b)

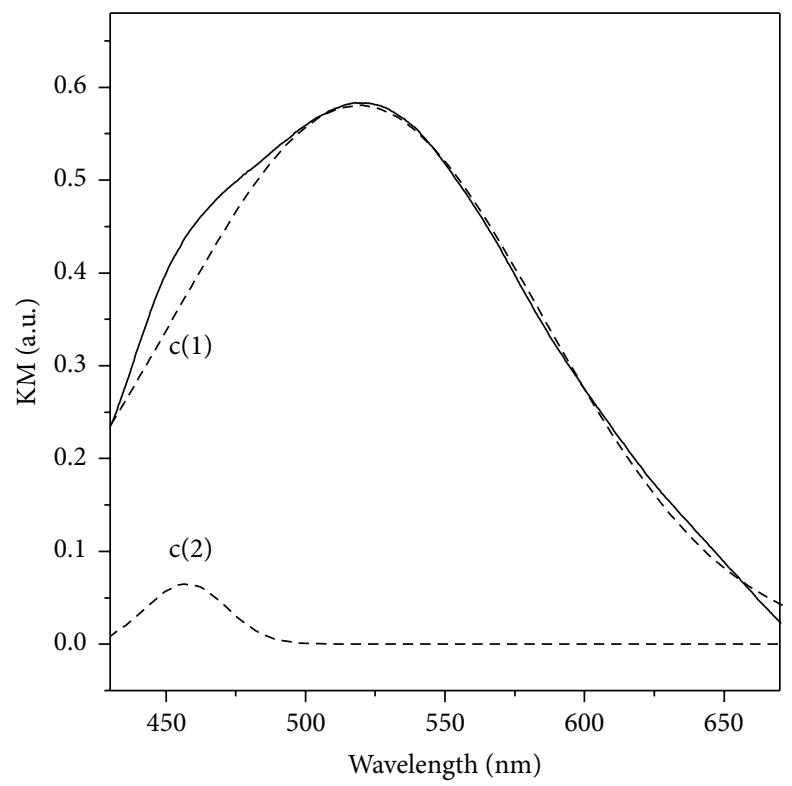

(c)

Figure 4: UV-vis spectra of VSB-5 and Au/VSB-5 composite (a). The difference spectra of Au/VSB-5 composite before (b) and after (c) the catalytic reaction with the background correction on that of VSB-5 after the Kubelka-Munk function analyses. The peaks were fitted by using Gaussian function.

the diffraction peak of $\mathrm{Au}$ (111) before and after the catalytic reaction from the enlargement of the XRD pattern could be found in the inset in Figure 2, which is due to the small size of gold crystals in the Au/VSB-5 composites according to the Scherrer formula.

HRTEM images of Au/VSB-5 before and after the first catalytic cycle are shown in Figure 3. One-dimensional ordered channels of VSB-5 nanocrystal could be apparently observed for both composites, indicating that the structure of VSB-5 was preserved after the catalytic reaction, which is in accordance with the XRD results. The gold particles in the channels may be observed directly in Figure 3(a). A little obscure morphology of gold particles is due to the low contrast between the nickel phosphate framework of VSB-5 


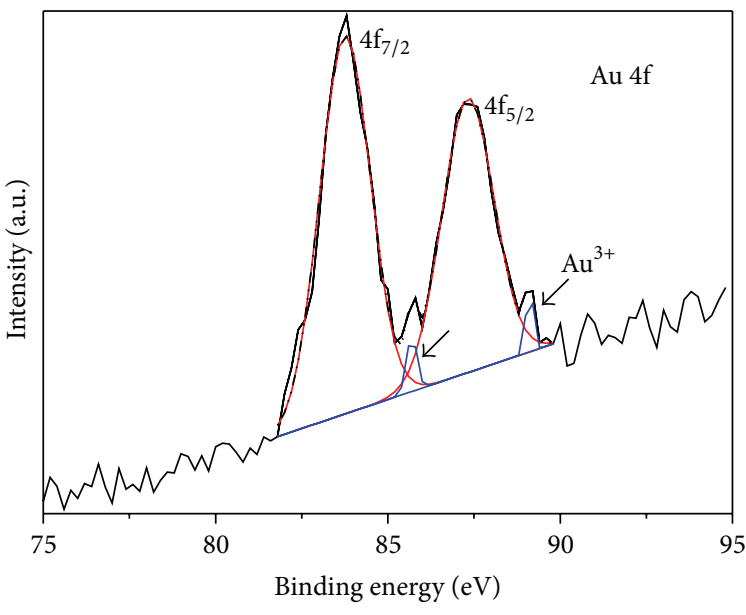

(a)

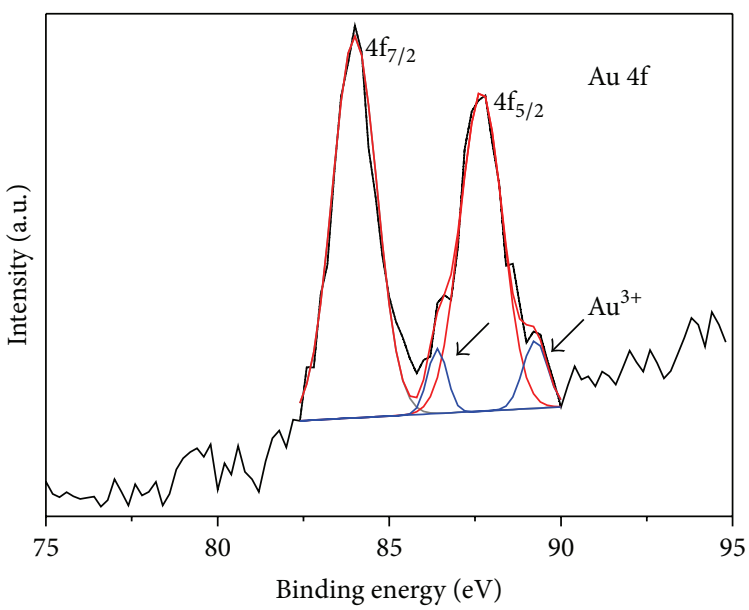

(b)

Figure 5: XPS spectra of Au 4f for Au/VSB-5 before (a) and after (b) the first cycle catalytic reaction with Gaussian peak fittings.

and gold [21]. Besides, only several gold particles with sizes of about $2-5 \mathrm{~nm}$ outside of the channels of VSB-5 could be found from the HRTEM image and this indicated that most of the gold nanocomposites were immobilized in the channels of VSB-5. After the first catalytic cycle, more gold particles with sizes about $2-5 \mathrm{~nm}$ were observed outside the channels (average size of $c a .3 .9 \mathrm{~nm}$ ). The contrast between the nickel phosphate framework of VSB-5 and gold became a little high, suggesting that these gold particles may have better crystallinity. Therefore, it can be deduced that some of the gold particles moved out of the channels and formed dispersed particles with suitable size due to the heating effects during the catalytic reaction. The gold particles formed by the heating effects have uniformed particle size suggesting that the dispersion of $\mathrm{Au}$ in the channels was very well. The loading amount of gold was low which limited further growth into larger particles. It was reported that the gold nanoparticles with sizes of 2-4 nm on other supports were dominantly responsible for the catalytic oxidation of $\mathrm{CO}[6$, 30]. The enhancement of the catalytic activity over Au/VSB5 after the first catalytic cycle may partly result from the well dispersed gold nanoparticles with suitable particle size in line with the result of $\mathrm{Au}$ on MOF [20]. Another possible reason for the enhancement may come from the increased crystallinity.

UV-vis spectra of Au/VSB-5 nanocomposites before and after the first catalytic cycle and VSB-5 are shown in Figure 4. A strong absorption band centered at $410 \mathrm{~nm}$ could be observed, which is due to $d$ - $d$ electron transition of $\mathrm{Ni}^{2+}$ in VSB-5 (Figure 4(a)). Figures 4(b) and 4(c) show the difference spectra of Au/VSB-5 and VSB-5 after the Kubelka-Munk function analyses. Obvious surface plasma resonance absorption peaks of gold nanoparticles may be observed and two absorption peaks centered at about 464 and 540/519 $\mathrm{nm}$ were observed after the peaking fitting using Gaussian method for both Au/VSB-5s before (Figure 4(b)) and after the catalytic reaction (Figure 4(c)). The absorption peaks centered at $464 \mathrm{~nm}$ (curve 1 in Figures 4(b) and 4(c)) were very similar to that of the reported $1.1 \mathrm{~nm} \mathrm{Au}$, indicating that this absorption may come from the contribution of gold nanoparticles inside the channels [26]. The broadened peaks centered at 540/519 nm (curve 2 in Figures 4(b) and $4(c)$ ) were attributed to the absorption of gold nanoparticles outside the channels of VSB-5 since they were larger particles than those inside the channels corresponding to their large red-shifts based on the Mie theory [31]. The position of this plasma peak shifted from $540 \mathrm{~nm}$ before the catalytic reaction to $519 \mathrm{~nm}$ after the catalytic reaction, suggesting that more small size gold nanoparticles formed, resulting in a little smaller average size of the gold outside of the channels after the reaction. This result is also in accordance with the following intensity analysis. After the first catalytic cycle, the absorption intensity of the gold nanoparticles outside the channels increased (curve 2 in Figure 4(c)) and the intensity of gold inside the channels decreased (curve 1 in Figure 4(c)), compared with that before the reaction (Figure 4(b)). Thus, it was further confirmed that some of the gold particles moved out of the channels and aggregated to form small particles during the catalytic reaction and these small gold particles contributed to the enhanced activity rather than the particles inside the channels.

XPS spectra of Au for Au/VSB-5 before and after the first cycle catalytic reaction are given in Figure 5. Two main peaks corresponding to $\mathrm{Au} 4 \mathrm{f}_{7 / 2}$ and $4 \mathrm{f}_{5 / 2}$ features are observed. The $\mathrm{Au}^{3+}$ ions and $\mathrm{Au}^{0}$ were identified by nonlinear Gaussian peak fittings method. The main peaks at $83.9 / 84.0$ and $87.7 / 87.7$ were attributed to the metallic Au before (Figure 5(a)) and after (Figure 5(b)) the catalytic reaction, respectively. The peaks at 86.1/86.4 and at 89.2/89.3 were assigned to the $\mathrm{Au}^{3+}$ ions, the same as the reported results $[10,32]$. No obvious shift was observed for the $\mathrm{Au} 4 \mathrm{f}$ peak positions after the catalytic reaction, indicating that the interaction between Au and VSB-5 hardly changed during the catalytic process. However, the intensities of peaks assigned to $\mathrm{Au}^{3+}$ that increased with the intensity (integrating peak area) ratio of $\mathrm{Au}^{0} / \mathrm{Au}^{3+}$ decreased from 36.8 to 10.6 after the first 
catalytic cycle for Au/VSB-5. Thus, the interaction did not play a main role in the enhancement of the catalytic activity, while the higher activity of Au/VSB-5 after the first cycle catalytic reaction may be also due to the increased ratio of $\mathrm{Au}^{3+} / \mathrm{Au}^{0}$, where $\mathrm{Au}^{3+}$ is related to the formation of $\mathrm{Au}^{\sigma+}$ $\mathrm{Au}^{0}[10]$.

\section{Conclusions}

Au/VSB-5 composite with Au loading amount of 1.43 wt.\% has been successfully prepared when choosing microporous VSB-5 as the support. This material possesses high catalytic activity for the $\mathrm{CO}$ oxidation reaction. The detailed factors influencing the property have been analyzed. A certain amount of gold nanoparticles stably existed outside the channels of VSB-5. The high $\mathrm{Au}^{3+} / \mathrm{Au}^{0}$ ratio rather than the interaction between $\mathrm{Au}$ and the support should be the main factors for the enhanced catalytic activity on Au/VSB-5 composite from the XPS analyses. This composite may also be used as powerful catalyst in other fields.

\section{Conflict of Interests}

The authors declare that there is no conflict of interests regarding the publication of this paper.

\section{Acknowledgments}

This work is financially supported by Public Projects of Zhejiang Province (no. 2014C31026), International S\&T Cooperation Program of China (no. 2013DFG52490), and the National Natural Science Foundation of China (no. 51372237).

\section{References}

[1] M. Ikegami, T. Matsumoto, Y. Kobayashi et al., "Air purification by gold catalysts supported on PET nonwoven fabric," Applied Catalysis B: Environmental, vol. 134-135, pp. 130-135, 2013.

[2] F. Menegazzo, M. Signoretto, F. Pinna et al., "Oxidative esterification of renewable furfural on gold-based catalysts: which is the best support?" Journal of Catalysis, vol. 309, pp. 241-247, 2014.

[3] M. Haruta, T. Kobayashi, H. Sano, and N. Yamada, "Novel gold catalysts for the oxidation of carbon monoxide at a temperature far below $0{ }^{\circ}$ C", Chemistry Letters, vol. 16, no. 2, pp. 405-408, 1987.

[4] Z. Chen, C. Della Pina, E. Falletta et al., "Facile synthesis of polyaniline using gold catalyst," Journal of Catalysis, vol. 259, no. 1, pp. 1-4, 2008.

[5] C. Della Pina, E. Falletta, L. Prati, and M. Rossi, "Selective oxidation using gold," Chemical Society Reviews, vol. 37, no. 9, pp. 2077-2095, 2008.

[6] J.-N. Lin and B.-Z. Wan, "Effects of preparation conditions on gold/Y-type zeolite for CO oxidation," Applied Catalysis B: Environmental, vol. 41, no. 1-2, pp. 83-95, 2003.

[7] Y. Iizuka, T. Miyamae, T. Miura, M. Okumura, M. Daté, and M. Haruta, "A kinetic study on the low temperature oxidation of CO over Ag-contaminated Au fine powder," Journal of Catalysis, vol. 262, no. 2, pp. 280-286, 2009.
[8] H. Wang, H. Zhu, Z. Qin, F. Liang, G. Wang, and J. Wang, "Deactivation of a $\mathrm{Au} / \mathrm{CeO}_{2}-\mathrm{Co}_{3} \mathrm{O}_{4}$ catalyst during $\mathrm{CO}$ preferential oxidation in $\mathrm{H}_{2}$-rich stream," Journal of Catalysis, vol. 264, no. 2, pp. 154-162, 2009.

[9] M. Haruta, "Size- and support-dependency in the catalysis of gold," Catalysis Today, vol. 36, no. 1, pp. 153-166, 1997.

[10] Z. Chen and Q. Gao, "Enhanced carbon monoxide oxidation activity over gold-ceria nanocomposites," Applied Catalysis B: Environmental, vol. 84, no. 3-4, pp. 790-796, 2008.

[11] X. Bokhimi, R. Zanella, and C. Angeles-Chavez, "Rutilesupported $\mathrm{Ir}, \mathrm{Au}$, and Ir-Au catalysts for $\mathrm{CO}$ oxidation," The Journal of Physical Chemistry C, vol. 114, no. 33, pp. 14101-14109, 2010.

[12] Q. Ye, J. Wang, J. Zhao et al., "Pt or Pd-doped $\mathrm{Au} / \mathrm{SnO}_{2}$ catalysts: high activity for low-temperature $\mathrm{CO}$ oxidation," Catalysis Letters, vol. 138, no. 1-2, pp. 56-61, 2010.

[13] W. Zhu, J. P. Zheng, R. Liang et al., "Ultra-low platinum loading high-performance PEMFCs using buckypaper-supported electrodes," Electrochemistry Communications, vol. 12, no. 11, pp. 1654-1657, 2010.

[14] Q. Li, Y. Zhang, G. Chen, J. Fan, H. Lan, and Y. Yang, "Ultralow-gold loading $\mathrm{Au} / \mathrm{CeO}_{2}$ catalysts for ambient temperature CO oxidation: Effect of preparation conditions on surface composition and activity," Journal of Catalysis, vol. 273, no. 2, pp. 167-176, 2010.

[15] R. Zhao, D. Ji, G. Lv et al., "A highly efficient oxidation of cyclohexane over Au/ZSM-5 molecular sieve catalyst with oxygen as oxidant," Chemical Communications, vol. 10, no. 7, pp. 904-905, 2004.

[16] G. Riahi, D. Guillemot, M. Polisset-Thfoin, A. A. Khodadadi, and J. Fraissard, "Preparation, characterization and catalytic activity of gold-based nanoparticles on HY zeolites," Catalysis Today, vol. 72, no. 1-2, pp. 115-121, 2002.

[17] M. T. Bore, M. P. Mokhonoana, T. L. Ward, N. J. Coville, and A. K. Datye, "Synthesis and reactivity of gold nanoparticles supported on transition metal doped mesoporous silica," Microporous and Mesoporous Materials, vol. 95, no. 1-3, pp. 118-125, 2006.

[18] B. Solsona, M. Conte, Y. Cong, A. Carley, and G. Hutchings, "Unexpected promotion of $\mathrm{Au} / \mathrm{TiO}_{2}$ by nitrate for $\mathrm{CO}$ oxidation," Chemical Communications, no. 18, pp. 2351-2353, 2005.

[19] J. Jiang, Q. Gao, and Z. Chen, "Gold nanocatalysts supported on protonic titanate nanotubes and titania nanocrystals," Journal of Molecular Catalysis A: Chemical, vol. 280, no. 1-2, pp. 233-239, 2008.

[20] H.-L. Jiang, B. Liu, T. Akita, M. Haruta, H. Sakurai, and Q. Xu, "Au@ZIF-8: CO oxidation over gold nanoparticles deposited to metal-organic framework," Journal of the American Chemical Society, vol. 131, no. 32, pp. 11302-11303, 2009.

[21] N. Guillou, Q. Gao, P. M. Forster et al., "Nickel(II) phosphate VSB-5: a magnetic nanoporous hydrogenation catalyst with 24ring tunnels," Angewandte Chemie International Edition, vol. 40, no. 15 , pp. 2831-2834, 2001.

[22] S. H. Jhung, J.-S. Chang, S.-E. Park, P. M. Forster, G. Férey, and A. K. Cheetham, "Template-free synthesis of the nanoporous nickel phosphate VSB-5 under microwave irradiation," Chemistry of Materials, vol. 16, no. 8, pp. 1394-1396, 2004.

[23] A. Samadi-Maybodi and S. K. H. Nejad-Darzi, "Phase-selective crystallization of nickel phosphate VSB-5 with (2-hydroxyethyl) trimethylammonium hydroxide as template," Crystal Research and Technology, vol. 46, no. 4, pp. 383-388, 2011. 
[24] D. Gao and Q. Gao, "Synthesis, characterization and catalytic properties in oxidation of styrene over cobalt-substituted microporous nickel phosphate CoVSB-5," Microporous and Mesoporous Materials, vol. 85, no. 3, pp. 365-373, 2005.

[25] M. N. Timofeeva, Z. Hasan, A. Y. Orlov et al., "Fe-containing nickel phosphate molecular sieves as heterogeneous catalysts for phenol oxidation and hydroxylation with $\mathrm{H}_{2} \mathrm{O}_{2}$," Applied Catalysis B: Environmental, vol. 107, pp. 197-204, 2011.

[26] C. Li, H. Kawada, X. Sun, H. Xu, Y. Yoneyama, and N. Tsubaki, "Highly efficient alcohol oxidation on nanoporous VSB-5 nickel phosphate catalyst functionalized by $\mathrm{NaOH}$ treatment," ChemCatChem, vol. 3, no. 4, pp. 684-689, 2011.

[27] Z. Chen, Q. Gao, D. Gao, Q. Wei, and M. Ruan, "Metallic Ag arrays assembled in nanoporous VSB- 5 nanocrystals by a simple method: a novel catalyst for the synthesis of olefin aldehyde from styrene," Materials Letters, vol. 60, no. 15, pp. 1816-1822, 2006.

[28] Z. Chen, Q. Gao, C. Wu, M. Ruan, and J. Shi, "Preparation and properties of an ordered, uniform $0.9 \mathrm{~nm} \mathrm{Ag}$ array assembled in a nanoporous VSB-1 by a simple soft chemical method," Chemical Communications, vol. 10, no. 17, pp. 1998-1999, 2004.

[29] Z. Chen, Q. Gao, and M. Ruan, "Electronic coupling onedimensional $\mathrm{Ag} / \mathrm{ZnS}$ nanocomposites in a nanoporous nickel phosphate host," Nanotechnology, vol. 18, no. 25, Article ID 255607, 2007.

[30] K.-C. Wu, Y.-L. Tung, Y.-L. Chen, and Y.-W. Chen, "Catalytic oxidation of carbon monoxide over gold/iron hydroxide catalyst at ambient conditions," Applied Catalysis B: Environmental, vol. 53, no. 2, pp. 111-116, 2004.

[31] T. G. Schaaff, M. N. Shafigullin, J. T. Khoury et al., "Isolation of smaller nanocrystal Au molecules: robust quantum effects in optical spectra," Journal of Physical Chemistry B, vol. 101, no. 40, pp. 7885-7891, 1997.

[32] B. C. Sih and M. O. Wolf, "Dielectric medium effects on collective surface plasmon coupling interactions in oligothiophenelinked gold nanoparticles," The Journal of Physical Chemistry B, vol. 110, no. 45, pp. 22298-22301, 2006. 

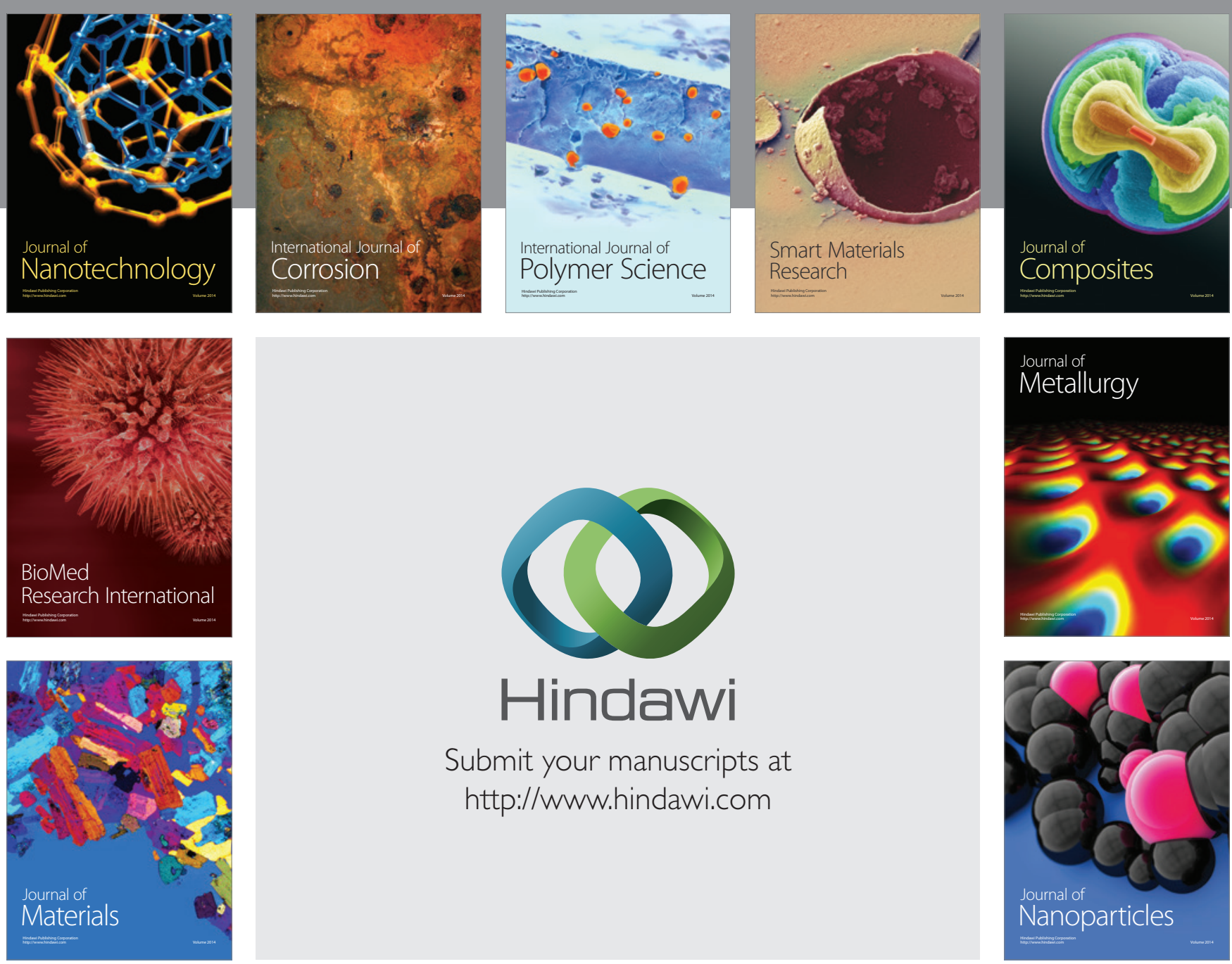

Submit your manuscripts at http://www.hindawi.com
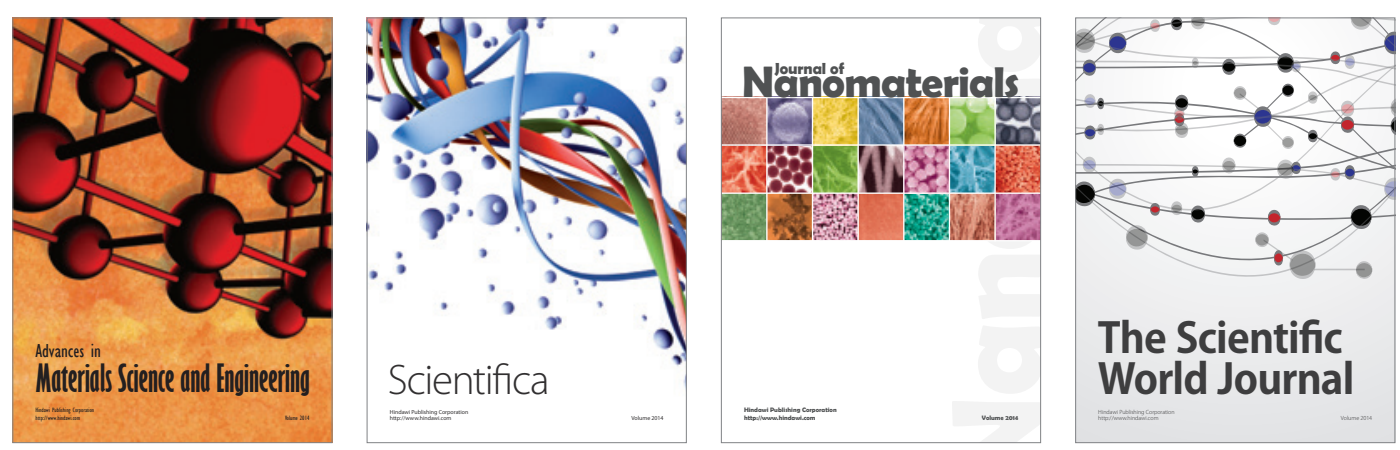

\section{The Scientific World Journal}
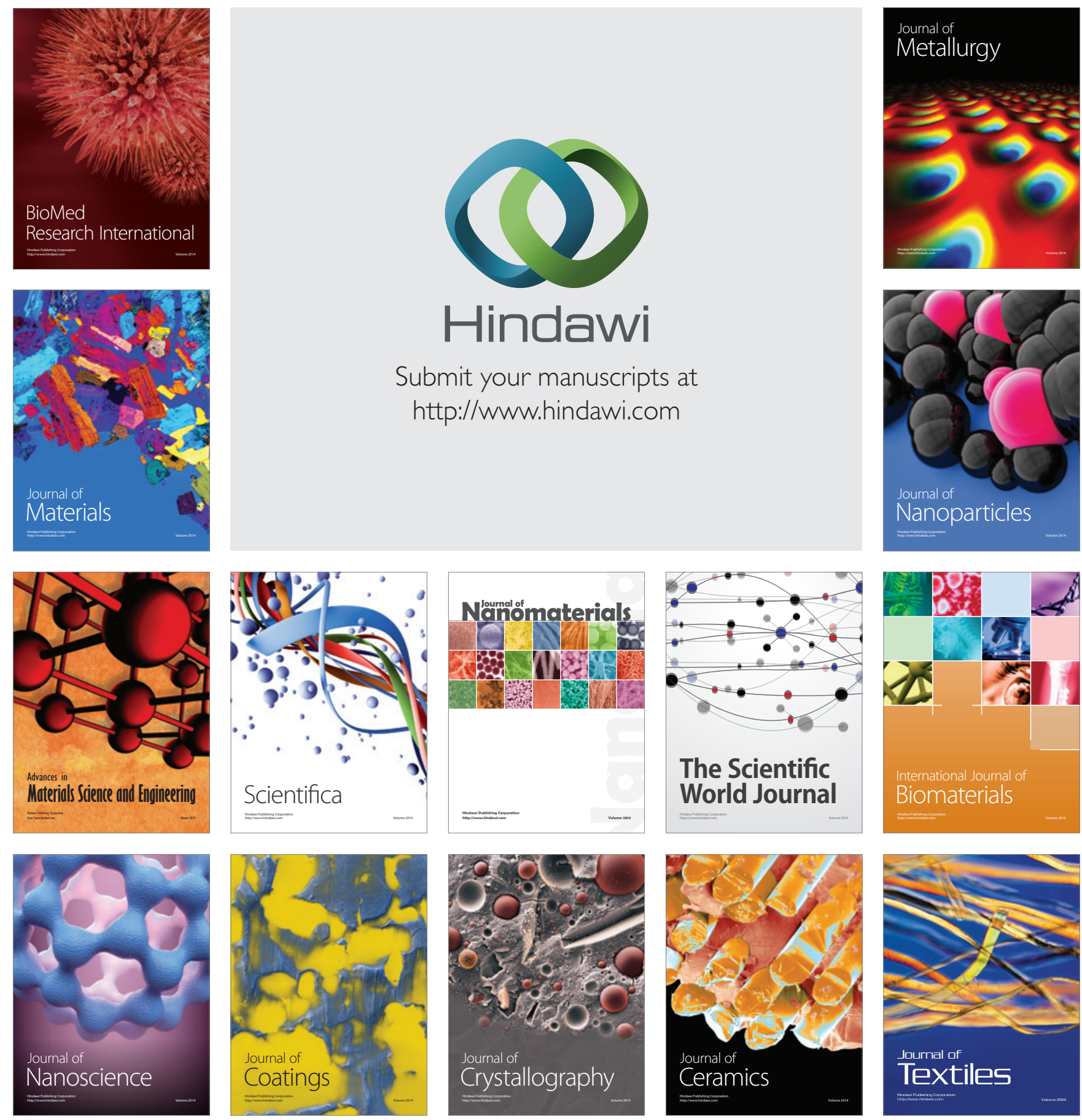